\title{
Investigation of Electron Beam Induced Mass Loss of Embedding Media in the Low Voltage STEM
}

\author{
Veronika Novotna $^{1,2}$, Kamila Hrubanova ${ }^{1,3}$, Jana Nebesarova ${ }^{4}$ and Vladislav Krzyzanek ${ }^{1}$ \\ 1. Institute of Scientific Instrument ASCR, Department of Electron Microscopy, Brno, Czech Republic. \\ Brno University of Technology, Department of Biomedical Engineering, Brno, Czech Republic. \\ 3. Brno University of Technology, Institute of Physical Engineering, Brno, Czech Republic. \\ 4. Biology center ASCR, Institute of Parasitology, Ceske Budejovice, Czech Republic.
}

The STEM (scanning transmission electron microscope) is useful device combining features of scanning and transmission electron microscopes. The sample in form of ultrathin section is scanned by the electron probe and the transmitted electrons are detected. Except the dedicated STEMs [1] this mode can exist as options in both TEM and SEM [2]. The STEM based on the SEM equipped by a transmission detector (often called as low voltage STEM) was used for presented experiments. Nowadays, the low voltage STEM is more often used, and in many cases replaces the typical TEM. Here, we report investigations of embedding media that are typically used for TEM preparation of biological samples.

The STEM detector may be able to detect both bright-field and dark-fields images (Figure 1). It uses much lower acceleration voltages $(30 \mathrm{kV}$ and below) than conventional TEM or dedicated STEM (60-300 kV). Moreover, biological samples suffer from the lack of contrast which is normally enhanced by staining with salts of heavy metals (lead citrate and uranyl acetate). Then, we face the problem if the stained structure is real or if it is only artefact. Thanks to low voltage STEM where the electrons have lower mean free electron path, the staining is not needed and the obtained images are more reliable. Possibility to achieve better contrast and also better resolution without staining and low dose imaging makes the STEM suitable for biological applications [3].

Materials like biological samples, polymers including embedding media are electron beam sensitive. There are few types of sample damages by the electron beam. Two the most important are the mass loss and the contamination. Both types of damages depend on the used electron energy and the electron dose applied to the sample. The mass loss depends on the sample composition and the contamination results from the poor vacuum in the specimen chamber of the SEM, cleanness of the sample surface, etc.

For simplicity, we have started with estimation of the mass loss of different types of embedding media for biological samples. We collected bright-field (BF) images of the same area at the sample; an overview of the scanned area taken after the last scan at a half magnification shows the increase of the signal due to the mass loss (Figure $2 \mathrm{a}$ ). The Figure $2 \mathrm{~b}$ shows the change of the BF signal to the total dose of electrons for all three embedding media. The experiments have been done using SEM Magellan 400L (FEI) at $30 \mathrm{kV}$ and a probe current of $1.6 \mathrm{pA}$. Samples in the form of $100 \mathrm{~nm}$ thin sections were cut from blocks of pure embedding media Epon, Spurr and LR White. The image processing was programmed in MATLAB.

Our results show that the mass loss at $30 \mathrm{kV}$ can be high even at a dose of $500 \mathrm{el} / \mathrm{nm}^{2}$, where for example, the LR white section was completely destroyed. The Epon resin in comparison with Spurr resin seems to be more stable under electron beam. At present the samples of different thicknesses are investigated using different microscope settings and STEM imaging modes. 


\section{References:}

[1] AV Crewe, J Wall and J Langmore, Science 168 (1970) p. 1338.

[2] A Bogner et al, Micron 38 (2007) p. 390.

[3] ND Browning et al in "The Application of Scanning Transmission Electron Microscopy (STEM) to the Study of Nanoscale Systems" (Springer, 2012) p. 11.

[4] The authors acknowledge the support by the grants CZ.1.07/2.3.00/20.0103 and LO1212 (EC and MEYS CR), 14-20012S (GACR) and TE01020118 (TACR).

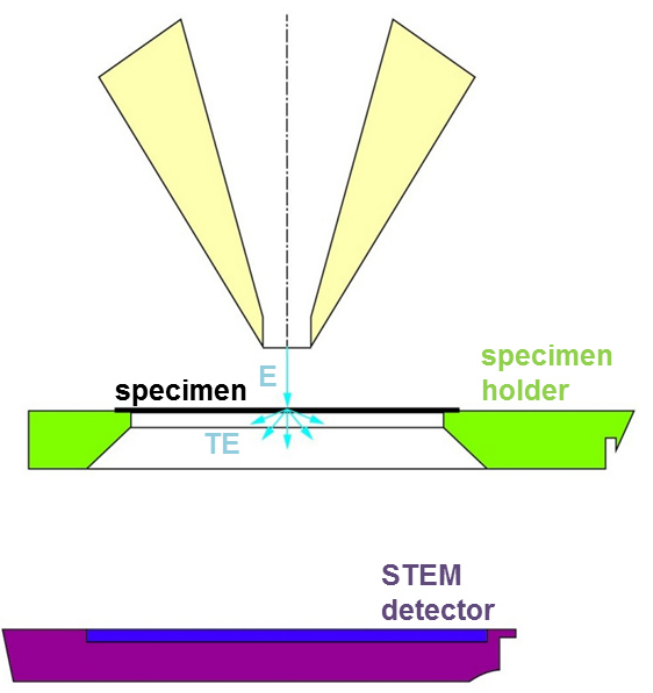

a

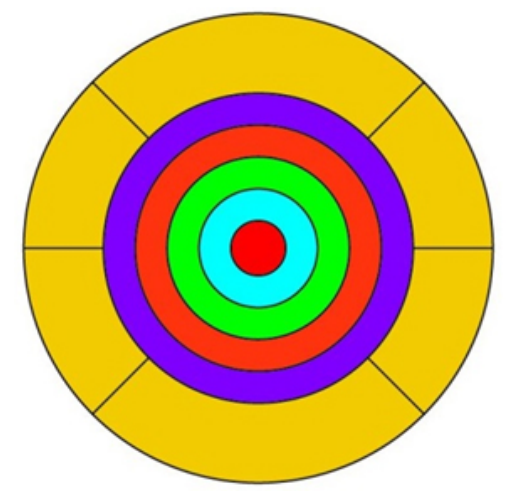

b

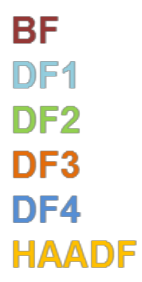

Figure 1. Schematic drawing of the transmission mode in SEM: (a) STEM detector location, (b) solid state STEM detector segments.

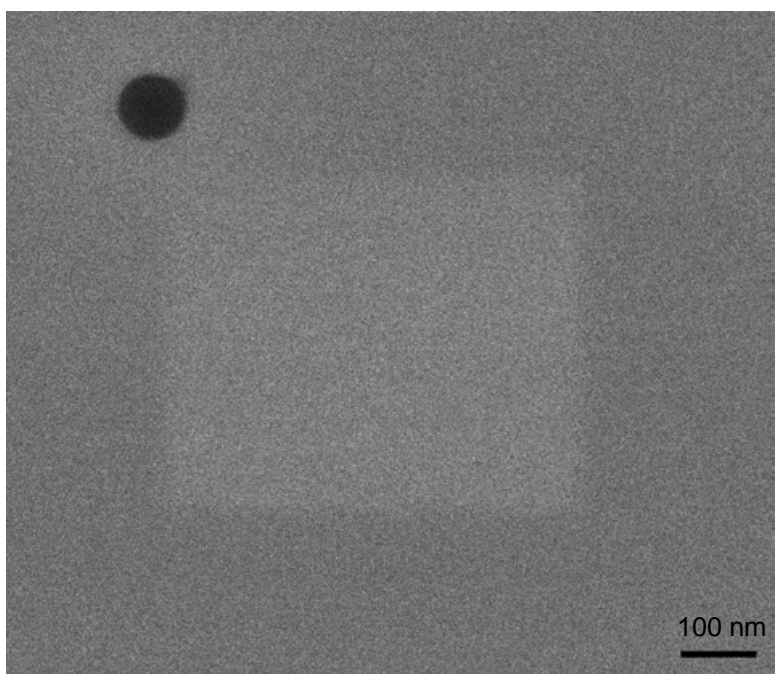

a

Figure 2. (a) Bright-field micrograph showing an overview of the many times scanned area taken after the last scan at a half magnification of Epon. (b) Mean value of the bright-field signal in the scanned image vs. total irradiated dose for LR White, Epon and Spurr. 